\title{
ON HALF INVERSE PROBLEM FOR DIFFERENTIAL PENCILS WITH THE SPECTRAL PARAMETER IN BOUNDARY CONDITIONS
}

\author{
S. A. BUTERIN
}

\begin{abstract}
A second-order differential pencil on a finite interval with spectral parameter dependent boundary conditions is considered. The inverse problem is studied of recovering the coefficients of the boundary value problem from its spectrum, provided that on one half of the interval they are known a priori. The uniqueness theorem for this inverse problem is proved and a constructive procedure for finding its solution is obtained.
\end{abstract}

\section{Introduction}

Consider the boundary value problem $L=L\left(q_{0}(x), q_{1}(x), h_{0}, h_{1}, H_{0}, H_{1}\right)$ of the form:

$$
\begin{aligned}
\ell y(x) & :=y^{\prime \prime}+\left(\rho^{2}-2 \rho q_{1}(x)-q_{0}(x)\right) y=0, \quad 0<x<\pi, \\
U(y) & :=y^{\prime}(0)-\left(h_{1} \rho+h_{0}\right) y(0)=0, \quad V(y):=y^{\prime}(\pi)+\left(H_{1} \rho+H_{0}\right) y(\pi)=0,
\end{aligned}
$$

where $\rho$ is a spectral parameter, $q_{j}(x) \in W_{1}^{j}[0,1]$ are complex-valued functions, $h_{j}, H_{j} \in \mathbb{C}, j=$ $0,1, h_{1} \neq \pm i, H_{1} \neq \pm i$. The latter conditions exclude from consideration Redge-type problems [1], which require separate investigation. Differential equations with a nonlinear dependence on the spectral parameter frequently appear in mathematics as well as in applications (see [2]-[6]). In this paper an inverse spectral problem for the pencil (1), (2) is studied. Inverse spectral problems consist in recovering operators from given their spectral characteristics. Such problems play an important role in mathematics and have many applications in natural sciences and engineering (see [7]-[12] and the references therein). Some aspects of inverse spectral problems for second order differential pencils were studied in [13]-[16] and other papers. In particular, in [16] it is proved that $L$ is uniquely determined by specifying its Weyl function, which is equivalent to specification of the spectra of two boundary value problems for equation (1) with one common boundary condition, and a constructive procedure for solving the inverse problem is given.

2000 Mathematics Subject Classification. Primary 421305, 42B08.

Key words and phrases. Differential pencils, half inverse problems, inverse spectral problems, Weyl function. 
In the present paper so-called half inverse spectral problem for the pencil $L$ is studied, which consists in recovering the coefficients of (1), (2) from its spectrum $\left\{\rho_{n}\right\}$, provided that they are known a priori on one half of the segment $[0, \pi]$. The uniqueness theorem for this inverse problem is proved, which in the case $h_{1}=H_{1}=0$ was proved in [17]. Here another approach is used, which is based on the interpolation of entire functions and gives a constructive procedure for solving the half inverse problem. Various aspects of the half inverse spectral problems for the Sturm-Liouville operator $\left(q_{1}(x) \equiv 0, h_{1}=H_{1}=0\right)$ were investigated in [18]-[23] and other works.

The paper is organized as follows. For the convenience of the reader in the next section some auxiliary assertions with brief proofs are provided. In Section 3 the uniqueness theorem is proved and a constructive procedure for solving the half inverse problem is given.

\section{Auxiliary assertions}

Let the functions $C(x, \rho), S(x, \rho), \varphi(x, \rho), \psi(x, \rho)$ and $\Phi(x, \rho)$ be solutions of equation (1) under the conditions

$$
\begin{aligned}
C(0, \rho) & =S^{\prime}(0, \rho)=\varphi(0, \rho)=\psi(\pi, \rho)=U(\Phi)=1, \\
C^{\prime}(0, \rho) & =S(0, \rho)=U(\varphi)=V(\psi)=V(\Phi)=0 .
\end{aligned}
$$

The functions $\Phi(x, \rho)$ and $M(\rho):=\Phi(0, \rho)$ are called respectively the Weyl solution and the Weyl function of the pencil $L$. For each fixed $x \in[0, \pi]$ the functions $C(x, \rho), S(x, \rho), \varphi(x, \rho)$, $\psi(x, \rho)$ together with their derivatives with respect to $x$ are entire in $\rho$. The eigenvalues $\rho_{n}$ of $L$ with account of multiplicity coincide with the zeros of its characteristic function $\Delta(\rho):=$ $\langle\psi(x, \rho), \varphi(x, \rho)\rangle$, where $\langle y, z\rangle:=y z^{\prime}-y^{\prime} z$. Clearly, $\Delta(\rho)=V(\varphi)=-U(\psi)$. The function $\Delta^{0}(\rho):=$ $\psi(0, \rho)$ is a characteristic function of the boundary value problem for equation (1) with the boundary conditions

$$
y(0)=V(y)=0 .
$$

We have

$$
\Phi(x, \rho)=-\frac{\psi(x, \rho)}{\Delta(\rho)}=S(x, \rho)+M(\rho) \varphi(x, \rho), \quad M(\rho)=-\frac{\Delta^{0}(\rho)}{\Delta(\rho)} .
$$

Let $\left\{\rho_{n}^{0}\right\}$ be the spectrum of (1), (3). Clearly, $\left\{\rho_{n}\right\} \cap\left\{\rho_{n}^{0}\right\}=\varnothing$. Thus, $M(\rho)$ is a meromorphic function with the poles $\rho_{n}$ and the zeros $\rho_{n}^{0}$. Denote

$$
Q(x):=\int_{0}^{x} q_{1}(t) d t
$$

Lemma 1. For $|\rho| \rightarrow \infty$ the following asymptotic representations hold:

$$
C(x, \rho)=\cos (\rho x-Q(x))+O\left(\frac{1}{\rho} \exp (|\operatorname{Im} \rho| x)\right),
$$




$$
\begin{aligned}
C^{\prime}(x, \rho) & =-\rho \sin (\rho x-Q(x))+O(\exp (|\operatorname{Im} \rho| x)), \\
S(x, \rho) & =\frac{\sin (\rho x-Q(x))}{\rho}+O\left(\frac{1}{\rho^{2}} \exp (|\operatorname{Im} \rho| x)\right), \\
S^{\prime}(x, \rho) & =\cos (\rho x-Q(x))+O\left(\frac{1}{\rho} \exp (|\operatorname{Im} \rho| x)\right)
\end{aligned}
$$

uniformly with respect to $x \in[0, \pi]$.

Proof. It is easy to check that the main parts of representations (5) and (7):

$$
\cos (\rho x-Q(x)), \quad \frac{\sin (\rho x-Q(x))}{\rho}
$$

form a fundamental system of solutions for the differential equation

$$
y^{\prime \prime}+\frac{q_{1}^{\prime}(x)}{\rho-q_{1}(x)} y^{\prime}+\left(\rho-q_{1}(x)\right)^{2} y=0 .
$$

Rewrite equation (1) in the form

$$
y^{\prime \prime}+\frac{q_{1}^{\prime}(x)}{\rho-q_{1}(x)} y^{\prime}+\left(\rho-q_{1}(x)\right)^{2} y=\left(q_{0}(x)+q_{1}^{2}(x)\right) y+\frac{q_{1}^{\prime}(x)}{\rho-q_{1}(x)} y^{\prime} .
$$

The function $y=C(x, \rho)$ is a solution of the Cauchy problem for equation (9) with the initial conditions $y(0)=1, y^{\prime}(0)=0$. By the method of variation of parameters we reduce this Cauchy problem to the integral equation

$C(x, \rho)=\cos (\rho x-Q(x))+\int_{0}^{x} \frac{\sin (\rho(x-t)-Q(x)+Q(t))}{\rho-q_{1}(t)}\left(\left(q_{0}(t)+q_{1}^{2}(t)\right) C(t, \rho)+\frac{q_{1}^{\prime}(t)}{\rho-q_{1}(t)} C^{\prime}(t, \rho)\right) d t$.

Differentiating (10) with respect to $x$ we get

$$
\begin{aligned}
C^{\prime}(x, \rho)= & -\left(\rho-q_{1}(x)\right)(\sin (\rho x-Q(x)) \\
& \left.-\int_{0}^{x} \frac{\cos (\rho(x-t)-Q(x)+Q(t))}{\rho-q_{1}(t)}\left(\left(q_{0}(t)+q_{1}^{2}(t)\right) C(t, \rho)+\frac{q_{1}^{\prime}(t)}{\rho-q_{1}(t)} C^{\prime}(t, \rho)\right) d t\right) .
\end{aligned}
$$

Denote

$$
\mu_{j}(\rho):=\max _{0 \leq x \leq \pi}\left(\left|C^{(j-1)}(x, \rho)\right| \exp (-|\operatorname{Im} \rho| x)\right), \quad j=1,2 .
$$

From (10), (11) we get

$$
\mu_{1}(\rho) \leq C\left(1+\frac{\mu_{1}(\rho)}{|\rho|}+\frac{\mu_{2}(\rho)}{|\rho|^{2}}\right), \quad \mu_{2}(\rho) \leq C\left(|\rho|+\mu_{1}(\rho)+\frac{\mu_{2}(\rho)}{|\rho|}\right),
$$

whence we obtain $\mu_{1}(\rho) \leq C, \mu_{2}(\rho) \leq C|\rho|$ or

$$
C(x, \rho)=O(\exp (|\operatorname{Im} \rho| x)), \quad C^{\prime}(x, \rho)=O(\rho \exp (|\operatorname{Im} \rho| x)), \quad|\rho| \rightarrow \infty .
$$


Substituting this into (10), (11) we arrive at (5), (6). The representations (7), (8) can be obtained analogously.

Put $G_{\delta}^{\beta, a}:=\{\rho:|\rho-\beta n-a| \geq \delta, n \in \mathbb{Z}\}, \delta>0$, and denote

$$
\sigma:=\frac{1}{2 \pi i} \ln \frac{i-H_{1}}{i+H_{1}}+\frac{Q(\pi)}{\pi} .
$$

Lemma 2. (i) For $|\rho| \rightarrow \infty$ the following asymptotic representations hold:

$$
\begin{gathered}
\psi(x, \rho)=\sqrt{1+H_{1}^{2}} \cos (\rho(\pi-x)+Q(x)-\pi \sigma)+O\left(\frac{1}{\rho} \exp (|\operatorname{Im} \rho|(\pi-x))\right), \\
\psi^{\prime}(x, \rho)=\rho \sqrt{1+H_{1}^{2}} \sin (\rho(\pi-x)+Q(x)-\pi \sigma)+O(\exp (|\operatorname{Im} \rho|(\pi-x)))
\end{gathered}
$$

uniformly with respect to $x \in[0, \pi]$.

Proof. Let $C_{1}(x, \rho), S_{1}(x, \rho)$ be solutions of equation (1) under the initial conditions

$$
C_{1}(\pi, \rho)=-S_{1}^{\prime}(\pi, \rho)=1, \quad C_{1}^{\prime}(\pi, \rho)=S_{1}(\pi, \rho)=0 .
$$

Thus,

$$
\psi(x, \rho)=C_{1}(x, \rho)+\left(H_{1} \rho+H_{0}\right) S_{1}(x, \rho) .
$$

According to Lemma 1 we have

$$
\begin{aligned}
& C_{1}(x, \rho)=\cos (\rho(\pi-x)-Q(\pi)+Q(x))+O\left(\frac{1}{\rho} \exp (|\operatorname{Im} \rho|(\pi-x))\right), \\
& S_{1}(x, \rho)=\frac{\sin (\rho(\pi-x)-Q(\pi)+Q(x))}{\rho}+O\left(\frac{1}{\rho^{2}} \exp (|\operatorname{Im} \rho|(\pi-x))\right),
\end{aligned}
$$

Formulae (15)-(17) give

or

$$
\begin{aligned}
\psi(x, \rho)= & \cos (\rho(\pi-x)-Q(\pi)+Q(x))+H_{1} \sin (\rho(\pi-x)-Q(\pi)+Q(x)) \\
& +O\left(\frac{1}{\rho} \exp (|\operatorname{Im} \rho|(\pi-x))\right)
\end{aligned}
$$

$$
\begin{aligned}
\psi(x, \rho)= & \sqrt{1+H_{1}^{2}}\left(\frac{1}{\sqrt{1+H_{1}^{2}}} \cos (\rho(\pi-x)-Q(\pi)+Q(x))\right. \\
& \left.+\frac{H_{1}}{\sqrt{1+H_{1}^{2}}} \sin (\rho(\pi-x)-Q(\pi)+Q(x))\right)+O\left(\frac{1}{\rho} \exp (|\operatorname{Im} \rho|(\pi-x))\right) .
\end{aligned}
$$

Since

$$
\cos \left(\frac{1}{2 i} \ln \frac{i-H_{1}}{i+H_{1}}\right)=\frac{1}{\sqrt{1+H_{1}^{2}}}, \quad \sin \left(\frac{1}{2 i} \ln \frac{i-H_{1}}{i+H_{1}}\right)=\frac{H_{1}}{\sqrt{1+H_{1}^{2}}}
$$


we arrive at (13). Analogously one can prove formula (14).

As in the proof of Lemma 2 we obtain

$$
\Delta(\rho)=\rho \sqrt{\left(1+h_{1}^{2}\right)\left(1+H_{1}^{2}\right)} \sin (\rho-\omega) \pi+O(\exp (|\operatorname{Im} \rho| \pi)),
$$

where

$$
\omega=\sigma+\frac{1}{2 \pi i} \ln \frac{i-h_{1}}{i+h_{1}} .
$$

By the standard method using (18) and Rouchè's theorem (see, e.g., [11]) one can show that the eigenvalues $\rho_{n}, n \in A:=\{n: n= \pm 0, \pm 1, \pm 2, \ldots\}$, have the form

$$
\rho_{n}=n+\omega+O\left(\frac{1}{n}\right), \quad|n| \rightarrow \infty .
$$

According to (20) for sufficiently large $|n|$ the eigenvalues $\rho_{n}$ are simple. By virtue of (19), (20) the specification of the spectrum (and of the number $h_{1}$ ) determines the value $\omega$ (and $\sigma$ ) up to an integer summand.

Using Hadamard's factorization theorem and the asymptotics (18) one can expand the characteristic function $\Delta(\rho)$ into an infinite product. More precisely, the following lemma holds.

Lemma 3. The characteristic function $\Delta(\rho)$ is determined up to a sign by the specification of its zeros and the numbers $h_{1}, H_{1}$. Moreover, the following representation holds:

$$
\Delta(\rho)=\sqrt{1+H_{1}^{2}} \tilde{\Delta}(\rho)
$$

where

$$
\tilde{\Delta}(\rho)=\sqrt{1+h_{1}^{2}}\left\{\begin{array}{l}
\sin \omega \pi \exp (-\pi \rho \operatorname{ctg} \omega \pi)\left(\rho_{-0}-\rho\right) \prod_{n=-\infty}^{\infty} \frac{\rho_{n}-\rho}{\rho_{n 0}} \exp \left(\frac{\rho}{\rho_{n 0}}\right), \omega \notin \mathbb{Z}, \\
(-1)^{\omega} \pi\left(\rho-\rho_{-0}\right)\left(\rho-\rho_{-\omega}\right) \prod_{\substack{n \neq-\omega \\
n=-\infty}}^{\infty} \frac{\rho_{n}-\rho}{\rho_{n 0}} \exp \left(\frac{\rho}{\rho_{n 0}}\right),
\end{array} \quad \omega \in \mathbb{Z},\right.
$$

and $\rho_{n 0}=n+\omega$.

Proof. According to Hadamard's factorization theorem we have

$$
\Delta(\rho)=C \exp \left(C_{1} \rho\right) \rho^{m} \prod_{\rho_{n} \neq 0}\left(1-\frac{\rho}{\rho_{n}}\right) \exp \left(\frac{\rho}{\rho_{n}}\right), \quad C, C_{1}-\text { const },
$$

where $m \geq 0$ is the multiplicity of the zero eigenvalue. Let for definiteness $\omega \notin \mathbb{Z}$. Consider the function $\Delta_{0}(\rho)=\rho \sin (\rho-\omega) \pi$, whose expansion has the form

$$
\Delta_{0}(\rho)=C^{0} \exp \left(C_{1}^{0} \rho\right) \rho \prod_{n=-\infty}^{\infty}\left(1-\frac{\rho}{\rho_{n 0}}\right) \exp \left(\frac{\rho}{\rho_{n 0}}\right),
$$


where

$$
C^{0}=\lim _{\rho \rightarrow 0} \frac{\Delta_{0}(\rho)}{\rho}=-\sin \omega \pi, \quad C_{1}^{0}=\lim _{\rho \rightarrow 0} \frac{d}{d \rho} \ln \frac{\Delta_{0}(\rho)}{\rho}=-\pi \operatorname{ctg} \omega \pi .
$$

Denote for briefness $D:=\sqrt{\left(1+h_{1}^{2}\right)\left(1+H_{1}^{2}\right)}$. According to (18) we have

$$
\frac{\Delta(\rho)}{\Delta_{0}(\rho)}=D+O\left(\frac{1}{\rho}\right), \quad \rho \in G_{\delta}^{1, \omega}, \quad|\rho| \rightarrow \infty .
$$

Together with (23), (24) this gives

$$
\begin{gathered}
C=(-1)^{m+1} D C^{0} \prod_{n \in A} \frac{p_{n}}{p_{n 0}}, \quad p_{n}=\left\{\begin{array}{c}
\rho_{n}, \rho_{n} \neq 0, \\
1, \rho_{n}=0,
\end{array} \quad p_{n 0}=\left\{\begin{array}{c}
n+\omega, n \in \mathbb{Z}, \\
1, \quad n=-0,
\end{array}\right.\right. \\
C_{1}=C_{1}^{0}+\sum_{n \in A}\left(r_{n 0}-r_{n}\right), \quad r_{n}=\left\{\begin{array}{rr}
\rho_{n}^{-1}, \rho_{n} \neq 0, \\
0, \quad \rho_{n}=0,
\end{array} \quad r_{n 0}=\left\{\begin{array}{cc}
(n+\omega)^{-1}, & n \in \mathbb{Z}, \\
0, & n=-0 .
\end{array}\right.\right.
\end{gathered}
$$

Formulae (23), (25)-(27) give (21), (22) for $\omega \notin \mathbb{Z}$. The case $\omega \in \mathbb{Z}$ is treated analogously.

\section{Half inverse problem}

Consider the following inverse problem.

Problem 1. Let the spectrum $\left\{\rho_{n}\right\}_{n \in A}$ of $L$ be given. Find $L$, provided that the numbers $h_{0}, h_{1}$ and the functions $q_{0}(x), q_{1}(x)$ on $(0, \pi / 2)$ are known a priori.

Let us prove the uniqueness theorem for Problem 1.

Theorem 1. The specification of the spectrum $\left\{\rho_{n}\right\}_{n \in A}$ determines $L$ uniquely, provided that the numbers $h_{0}, h_{1}$ and the functions $q_{0}(x), q_{1}(x)$ on $(0, \pi / 2)$ are known a priori. Thus, the specification of $\left\{\rho_{n}\right\}_{n \in A}$ uniquely determines $L$, provided that (1), (2) are known a priori on one half of the segment $[0, \pi]$.

Proof. The formulae (4) give

$$
\psi(x, \rho)=\Delta^{0}(\rho) \varphi(x, \rho)-\Delta(\rho) S(x, \rho) .
$$

Denote

$$
\begin{gathered}
\Delta_{1}(\rho):=-\psi^{\prime}\left(\frac{\pi}{2}, \rho\right), \quad \Delta_{1}^{0}(\rho):=\psi\left(\frac{\pi}{2}, \rho\right), \\
\Xi(\rho):=\varphi^{\prime}\left(\frac{\pi}{2}, \rho\right), \quad \Theta(\rho):=\varphi\left(\frac{\pi}{2}, \rho\right) .
\end{gathered}
$$

We note that $\Delta_{1}(\rho)$ is a characteristic function of the boundary value problem

$$
\ell y(x)=0, \quad \frac{\pi}{2}<x<\pi, \quad y^{\prime}\left(\frac{\pi}{2}\right)=V(y)=0
$$


The function

$$
M_{1}(\rho):=-\frac{\Delta_{1}^{0}(\rho)}{\Delta_{1}(\rho)}
$$

is the Weyl function for (31). The functions $\Xi(\rho)$ and $\Theta(\rho)$ are, in turn, the characteristic functions of the boundary value problems

$$
\begin{aligned}
\ell y(x)=0, \quad 0<x<\frac{\pi}{2}, \quad U(y)=y^{\prime}\left(\frac{\pi}{2}\right)=0, \\
\ell y(x)=0, \quad 0<x<\frac{\pi}{2}, \quad U(y)=y\left(\frac{\pi}{2}\right)=0
\end{aligned}
$$

respectively. Let $\left\{\xi_{n}\right\}_{n \in A}$ and $\left\{\theta_{n}\right\}_{n \in \mathbb{Z}}$ be the spectra of the boundary value problems (33) and (34), i.e. they are the sequences of zeros of the functions $\Xi(\rho)$ и $\Theta(\rho)$ respectively. Thus, we have

$$
\xi_{n}=2 n+\omega_{1}+O\left(\frac{1}{n}\right), \quad \theta_{n}=2 n+\omega_{1}^{0}+O\left(\frac{1}{n}\right), \quad n \rightarrow \pm \infty .
$$

Let $m_{n}$ and $m_{n}^{0}$ be the multiplicities of the zeros $\xi_{n}$ and $\theta_{n}$ respectively. By virtue of (35) we have $m_{n}=m_{n}^{0}=1$ for sufficiently large $|n|$. Choose subsets of indexes $S \subset A, S_{0} \subset \mathbb{Z}$ so that the subsequences $\left\{\xi_{n}\right\}_{n \in S}$ and $\left\{\theta_{n}\right\}_{n \in S_{0}}$ contain all zeros of the functions $\Xi(\rho), \Theta(\rho)$ without multiplicity.

Put

$$
\tilde{\Delta}^{0}(\rho):=\frac{\Delta^{0}(\rho)}{\sqrt{1+H_{1}^{2}}}, \quad \tilde{\Delta}_{1}(\rho):=\frac{\Delta_{1}(\rho)}{\sqrt{1+H_{1}^{2}}}, \quad \tilde{\Delta}_{1}^{0}(\rho):=\frac{\Delta_{1}^{0}(\rho)}{\sqrt{1+H_{1}^{2}}} .
$$

According to (28)-(30), (36) we have

$$
\left.\begin{array}{r}
\tilde{\Delta}_{1}^{0}(\rho)=\tilde{\Delta}^{0}(\rho) \Theta(\rho)-\tilde{\Delta}(\rho) S(\pi / 2, \rho), \\
-\tilde{\Delta}_{1}(\rho)=\tilde{\Delta}^{0}(\rho) \Xi(\rho)-\tilde{\Delta}(\rho) S^{\prime}(\pi / 2, \rho) .
\end{array}\right\}
$$

According to (36) and Lemma 2 we get

$$
\left.\begin{array}{l}
d(\rho):=\tilde{\Delta}_{1}(\rho)+\rho \sin \left(\frac{\rho \pi}{2}+Q\left(\frac{\pi}{2}\right)-\pi \sigma\right)=O\left(\exp \left(\frac{|\operatorname{Im} \rho| \pi}{2}\right)\right), \\
d_{0}(\rho):=\tilde{\Delta}_{1}^{0}(\rho)-\cos \left(\frac{\rho \pi}{2}+Q\left(\frac{\pi}{2}\right)-\pi \sigma\right)=O\left(\frac{1}{\rho} \exp \left(\frac{|\operatorname{Im} \rho| \pi}{2}\right)\right) .
\end{array}\right\}
$$

Using the known method (see, e.g., [11]) one can prove the following estimates for sufficiently large $|\rho|$ :

$$
|\Theta(\rho)| \geq C_{\delta} \exp \left(\frac{|\operatorname{Im} \rho| \pi}{2}\right), \rho \in G_{\delta}^{2, \omega_{1}^{0}}, \quad|\Xi(\rho)| \geq C_{\delta}|\rho| \exp \left(\frac{|\operatorname{Im} \rho| \pi}{2}\right), \rho \in G_{\delta}^{2, \omega_{1}} .
$$

Lemma 4. The specification of the numbers $\left\{\xi_{n}\right\}_{n \in A},\left\{\theta_{n}\right\}_{n \in \mathbb{Z}}$,

$$
\left\{d^{(v)}\left(\xi_{n}\right)\right\}_{v=\overline{0, m_{n}-1}, n \in S}, \quad\left\{d_{0}^{(v)}\left(\theta_{n}\right)\right\}_{v=0, m_{n}^{0}-1}, n \in S_{0},
$$


and the values $Q(\pi / 2), \sigma$ uniquely determines the functions $\tilde{\Delta}_{1}(\rho), \tilde{\Delta}_{1}^{0}(\rho)$ by the formulae

$$
\left.\begin{array}{c}
\tilde{\Delta}_{1}(\rho)=-\rho \sin \left(\frac{\rho \pi}{2}+Q\left(\frac{\pi}{2}\right)-\pi \sigma\right)+d(\rho), \\
\tilde{\Delta}_{1}^{0}(\rho)=\cos \left(\frac{\rho \pi}{2}+Q\left(\frac{\pi}{2}\right)-\pi \sigma\right)+d_{0}(\rho),
\end{array}\right\}
$$

where

$$
\left.\begin{array}{rl}
d(\rho) & =\sum_{n \in A} d\left(\xi_{n}\right) \frac{\Xi(\rho)}{\left(\rho-\xi_{n}\right) \Xi^{\prime}\left(\xi_{n}\right)}, \\
d_{0}(\rho) & =\sum_{n \in \mathbb{Z}} d_{0}\left(\theta_{n}\right) \frac{\Theta(\rho)}{\left(\rho-\theta_{n}\right) \Theta^{\prime}\left(\theta_{n}\right)},
\end{array}\right\}
$$

if all zeros of the functions $\Theta(\rho), \Xi(\rho)$ are simple (the case of multiple zeros requires minor modifications).

Proof. It is sufficient to prove (42). The function

$$
\Xi_{n}(\rho):=\frac{\Xi(\rho)}{\rho-\xi_{n}}
$$

is entire after removing the singularity in $\xi_{n}$, and $\Xi_{n}\left(\xi_{n}\right)=\Xi^{\prime}\left(\xi_{n}\right)$. Consider the contour $\gamma_{\delta, n}:=$ $\left\{\rho:\left|\rho-\xi_{n}\right|=\delta\right\}, \delta>0$. For large $|n|$ the maximum modulus principle gives

$$
\left|\Xi^{\prime}\left(\xi_{n}\right)\right| \geq \min _{\rho \in \gamma_{\delta, n}}\left|\Xi_{n}(\rho)\right|=\frac{1}{\delta} \min _{\rho \in \gamma_{\delta, n}}|\Xi(\rho)| .
$$

By virtue of (39) $\left|\Xi^{\prime}\left(\xi_{n}\right)\right| \geq C\left|\xi_{n}\right|$. Moreover, according to (38) $\left|d\left(\xi_{n}\right)\right| \leq C$. Thus, the series in the first formula of (42) converges uniformly in bounded subsets of $\mathbb{C}$. Denote

$$
d_{1}(\rho):=\sum_{n \in A} d\left(\xi_{n}\right) \frac{\Xi(\rho)}{\left(\rho-\xi_{n}\right) \Xi^{\prime}\left(\xi_{n}\right)}, \quad \alpha(\rho):=\frac{d(\rho)-d_{1}(\rho)}{\Xi(\rho)} .
$$

After removing the singularities the function $\alpha(\rho)$ is entire in $\rho$. Using (38), (39) we get

$$
\frac{d(\rho)}{\Xi(\rho)}=O\left(\frac{1}{\rho}\right), \quad \rho \in G_{\delta}^{2, \omega_{1}}, \quad|\rho| \rightarrow \infty
$$

Moreover, we have

$$
\left|\frac{d_{1}(\rho)}{\Xi(\rho)}\right| \leq \sum_{n \in A} \frac{\left|d\left(\xi_{n}\right)\right|}{\left|\rho-\xi_{n}\right|\left|\Xi^{\prime}\left(\xi_{n}\right)\right|} \leq C\left(\sum_{n \in A} \frac{1}{\left|\rho-\xi_{n}\right|^{2}}\right)^{\frac{1}{2}} \leq C_{1}, \quad \rho \in G_{\delta}^{2, \omega_{1}},
$$

and

$$
\frac{d_{1}(i \tau)}{\Xi(i \tau)}=o(1), \quad \tau \in \mathbb{R}, \quad|\tau| \rightarrow \infty .
$$

Using the maximum modulus principle and Liouville's theorem we get $\alpha(\rho) \equiv 0$, and the first formula in (42) is proved. The second formula can be proved analogously. 
Let us return to the proof of Theorem 1. Using the given numbers $h_{0}, h_{1}$ and the functions $q_{0}(x), q_{1}(x), x \in(0, \pi / 2)$, we find the value $Q(\pi / 2)$, the functions $S(\pi / 2, \rho), S^{\prime}(\pi / 2, \rho)$ and the functions $\Xi(\rho), \Theta(\rho)$ by formulae (30). Then using the given spectrum $\left\{\rho_{n}\right\}_{n \in A}$ we find the number $\omega$ from the asymptotics (20) and construct the function $\tilde{\Delta}(\rho)$ by formula (22). Find the number $\sigma$ by formula (19). According to (37), (38) we have

$$
\begin{aligned}
& d^{(v)}\left(\xi_{n}\right)=\left.\frac{d^{v}}{d \rho^{v}}\left(\tilde{\Delta}(\rho) S^{\prime}\left(\frac{\pi}{2}, \rho\right)+\rho \sin \left(\frac{\rho \pi}{2}+Q\left(\frac{\pi}{2}\right)-\pi \sigma\right)\right)\right|_{\rho=\xi_{n}}, \\
& d_{0}^{(v)}\left(\theta_{n}\right)=-\left.\frac{d^{v}}{d \rho^{v}}\left(\tilde{\Delta}(\rho) S\left(\frac{\pi}{2}, \rho\right)+\cos \left(\frac{\rho \pi}{2}+Q\left(\frac{\pi}{2}\right)-\pi \sigma\right)\right)\right|_{\rho=\theta_{n}} .
\end{aligned}
$$

Formulae (32), (36) give

$$
M_{1}(\rho):=-\frac{\tilde{\Delta}_{1}^{0}(\rho)}{\tilde{\Delta}_{1}(\rho)} .
$$

According to Lemma 4 and formula (45) the function $M_{1}(\rho)$ is uniquely determined by specifying the given data. Note that the numbers $\omega$ and $\sigma$ are determined up to an integer sum-

mand and hence the functions $\tilde{\Delta}_{1}(\rho), \tilde{\Delta}_{1}^{0}(\rho)$ are determined up to a sign while their ratio (45) is determined uniquely. According to the uniqueness theorem in [16] the specification of $M_{1}(\rho)$ uniquely determines the numbers $H_{0}, H_{1}$ and the functions $q_{0}(x), q_{1}(x)$ on $(\pi / 2, \pi)$.

The proof of Theorem 1 is constructive and gives the following algorithm for solving the half inverse problem. Let the spectrum $\left\{\rho_{n}\right\}_{n \in A}$, the numbers $h_{0}, h_{1}$ and the functions $q_{0}(x)$, $q_{1}(x)$ on $(0, \pi / 2)$ be given.

\section{Algorithm 1.}

(i) Find the number $\omega$ from the asymptotics (20) by the formula

$$
\omega=\lim _{n \rightarrow \infty}\left(\rho_{n}-n\right)
$$

and calculate the function $\tilde{\Delta}(\rho)$ by formula (22);

(ii) construct the functions $\Xi(\rho), \Theta(\rho)$ by (30) and find their zeros $\left\{\xi_{n}\right\}_{n \in A},\left\{\theta_{n}\right\}_{n \in \mathbb{Z}}$;

(iii) find the numbers (40) by formulae (43), (44);

(iv) construct the functions $\tilde{\Delta}_{1}(\rho), \tilde{\Delta}_{1}^{0}(\rho)$ by formula (41);

(v) calculate the Weyl function $M_{1}(\rho)$ of the boundary value problem (31) by formula (45) and find the functions $q_{0}(x), q_{1}(x)$ on $(\pi / 2, \pi)$ and the coefficients $H_{0}, H_{1}$, using the algorithm in [16].

\section{Acknowledgements}

This research was supported in part by Russian Foundation for Basic Research and Taiwan National Science Council (projects 10-01-00099 and 10-01-92001- NSC-a) and by Mikhail Lomonosov Program of the Ministry of Education and Science of Russia and DAAD (project 5007). 


\section{References}

[1] V. A. Yurko, On boundary value problems with the parameter in the boundary conditions, Izvestjya AN SSR. Ser. Matem., 19 (1984), 398-409.

[2] J. D. Tamarkin, On Some Problems of the Theory of Ordinary Linear Differential Equations, Petrograd, 1917.

[3] M. V. Keldysh, On eigenvalues and eigenfunctions of some classes of non-selfadjoint equations, Dokl. Akad. Nauk SSSR, 77 (1951), 11-14.

[4] A. G. Kostyuchenko and A. A. Shkalikov, Selfadjoint quadratic operator pencils and elliptic problems, Funktional. Anal. i Prilozhen, 17 (1983), 38-61; English transl.: Funct. Anal. Appl., 17 (1983), 109-128.

[5] A. S. Markus, Introduction to the spectral theory of polynomial operator pencils, Shtinitsa, Kishinev, 1986; English transl., AMS, Providence, RI, 1988.

[6] V. A. Yurko, An inverse problem for systems of differential equations with nonlinear dependence on the spectral parameter, Diff. Uravneniya, 33 (1997), 390-395; English transl., Diff. Equations, 33 (1997), 388-394.

[7] V. A. Marchenko, Sturm-Liouville Operators and Their Applications, Naukova Dumka, Kiev, 1977; English transl., Birkhäuser, 1986.

[8] B. M. Levitan, Inverse Sturm-Liouville Problems, Nauka, Moscow, 1984; English transl., VNU Sci.Press, Utrecht, 1987.

[9] J. R. McLaughlin, Analytical methods for recovering coefficients in differential equations from spectral data, SIAM Rev., 28 (1986), 53-72.

[10] K. Chadan, D. Colton, L. Päivärinta and W. Rundell, An Introduction to Inverse Scattering and Inverse Spectral Problems. SIAM Monographs on Mathematical Modeling and Computation, SIAM, Philadelphia, PA, 1997.

[11] G. Freiling and V. A. Yurko, Inverse Sturm-Liouville Problems and Their Applications, NOVA Science Publishers, New York, 2001.

[12] V. A. Yurko, Method of Spectral Mappings in the Inverse Problem Theory. Inverse and Ill-posed Problems Series, VSP, Utrecht, 2002.

[13] M. G. Gasymov and G. Sh. Gusejnov, Determination of a diffusion operator from spectral data, Akad. Nauk Azerb. SSR Dokl., 37(1981), 19-23.

[14] M. Yamamoto, Inverse eigenvalue problem for a vibration of a string with viscous drag, J. Math. Anal. Appl., 152 (1990), 20-34.

[15] V. A. Yurko, An inverse problem for pencils of differential operators, Matem. Sbornik, 191 (2000), 137-160; English transl., Sbornik: Mathematics, 191 (2000), 1561-1586.

[16] S. A. Buterin, V. A. Yurko, Inverse spectral problem for pencils of differential operators on a finite interval, Vestnik Bashkir. Univ. (2006) no.4, 8-12.

[17] S. A. Buterin and C.-T. Shieh, Incomplete inverse spectral and nodal problems for differential pencils, Results in Mathematics, DOI: 10.1007/s00025-011-0137-6.

[18] C.-T. Shieh and V. A. Yurko, Inverse nodal and inverse spectral problems for discontinuous boundary value problems, J. Math. Anal. Appl., 374 (2008), 266-272.

[19] H. Hochschtadt and B. Liebermann, An inverse Sturm-Liouville problem with mixed given data, SIAM J. Appl. Math., 34 (1978), 676-680.

[20] F. Gesztesy and B. Simon, Inverse spectral analysis with partial information on the potential. II, The case of discrete spectrum, Trans. Amer. Math. Soc., 352 (2000), 2765-2787.

[21] L. Sakhnovich, Half-inverse problem on the finite interval, Inverse Problems, 17 (2001), 527-532.

[22] R. O. Hryniv and Y. V. Mykytyuk, Half-inverse spectral problems for Sturm-Liouville operators with singular potentials, Inverse Problems, 20 (2004), 1423-1444.

[23] O. Martinyuk and V. Pivovarchik, On the Hochstadt-Lieberman theorem, Inverse Problems, 26 (2010), 035011 (6pp).

Department of Mathematics and Mechanics, Saratov State University, Russian Federation.

E-mail: buterinsa@info.sgu.ru 